\title{
Cirsoid Aneurysm of the Scalp: A Case Report
}

\author{
Mayur Vinod Barhate ${ }^{1}$ Sanjay Shantilal Vhora ${ }^{2}$ Rohit Kumar Pandey ${ }^{1}$ \\ ${ }^{1}$ Department of General Surgery, B.J. Medical College and Sassoon \\ General Hospital, Pune, India \\ 2 Department of Neurosurgery, B.J. Medical College and Sassoon \\ General Hospital, Pune, India \\ Address for correspondence Mayur Vinod Barhate, MS, 102, Kiran \\ Songir, Near DSK Vidyanagri, Pashan-Sus Road, Baner, Pune 411045, \\ India (e-mail: mayur_rn2006@yahoo.co.in).
}

Indian J Neurosurg 2016;5:129-132.

\begin{abstract}
Cirsoid aneurysms (arteriovenous malformations [AVMs]) of the scalp are rarely encountered in neurosurgical practice. Patients with AVM of the scalp present clinically at times with headache and a small innocuous-looking subcutaneous scalp lump or a large, pulsatile mass with or without bruit, which has a propensity to massive hemorrhage. Complex vascular anatomy and interconnections, high shunt flow, and possible cosmetic complications tend to make their management difficult. We report a rare case of young man who presented with progressively enlarging swelling over left fronto-temporo-parietal and occipital region of the scalp since 8 years. Digital subtraction angiography of internal and external carotid vessels revealed high-flow, complex left parietal scalp AVM supplied by multiple tortuous

Keywords

- arteriovenous malformation

- cirsoid aneurysm

- scalp feeders from left superficial temporal artery (STA), left middle meningeal artery, left occipital artery with collateral supply from right occipital artery, and right STA. Drainage into external jugular vein through multiple tortuous frontal, temporal, and occipital scalp veins with venous varix. Being high-flow shunt and because of suspected cosmetic complications, surgical excision was considered.
\end{abstract}

\section{Introduction}

Cirsoid aneurysms of the scalp are rare lesion. It is an abnormal fistulous connection between the feeding arteries and draining veins without an intervening capillary bed. Various names being used to describe the vascular malformations of the scalp include cirsoid aneurysm, aneurysma serpentinum, aneurysm racemosum, plexiform angioma, arteriovenous fistula, and arteriovenous malformation (AVM). They are usually congenital in etiology; however, traumatic fistulas have also been described. These lesions present as innocuous looking small lump or a large pulsatile mass with propensity to massive hemorrhage. The draining veins often are dilated owing to the high velocity of blood flow through the fistulae. Complex aberrant vascular anatomy and high shunt flow tend to complicate the management of scalp AVM. The indications for treatment are cosmetic,

received

December 29, 2015

accepted

January 14, 2016

published online

June 8,2016
DOI http://dx.doi.org/

10.1055/s-0036-1581974. ISSN 2277-954X. relief of the pulsatile headache and tinnitus, and prevention of hemorrhage. Surgical excision has been the most common method of dealing with the aneurysm and is the treatment of choice. In this case report, we describe our experience with the surgical management of this complex lesion.

\section{Case Report}

A young man presented with a history of slowly progressive swelling over the left parieto-occipital region for the past 8 years ( - Fig. $\mathbf{1}$ ). It was associated with occasional headache. Initially, the patient noticed small swelling over the left parietal region of scalp 8 years back following some trauma to head. The swelling then increased gradually to involve whole of the left fronto-temporo-parietal and occipital region. He also gives a history of some prior surgical procedure of the lesion 2 years back but no documents were available. (c) 2016 Neurological Surgeons' Society of India
License terms

(요 (1) $\Theta$ 


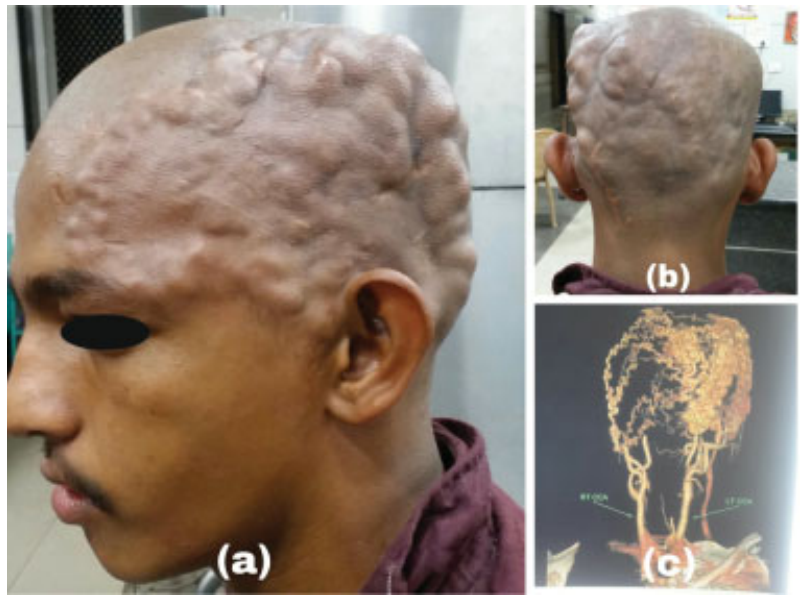

Fig. 1 Side view (a) and rear view (b) pre-op photographs of the patient. Pre-op digital subtraction angiography image (c).

General and systemic examinations were unremarkable with no focal neurological deficit. Local examination revealed large serpentine, irregular, soft, compressible, pulsatile scalp mass involving the whole left frontotemporo-parietal and occipital region with large tortuous dilated vessels. Easily palpable thrill and audible bruit were present. Digital subtraction angiography showed high-flow, complex left parietal scalp AVM supplied by multiple tortuous feeders from left superficial temporal artery, left middle meningeal artery, and left occipital artery with collateral supply from right superficial temporal artery and right occipital artery. Drainage into external jugular vein through multiple tortuous frontal, temporal, and occipital scalp veins with venous varix. There was no evidence of communication with intracranial circulation (-Fig. 2).

En block surgical excision of the lesion was planned. The patient was positioned with head elevated 30 degrees above the heart level. A question mark scalp flap was planned.

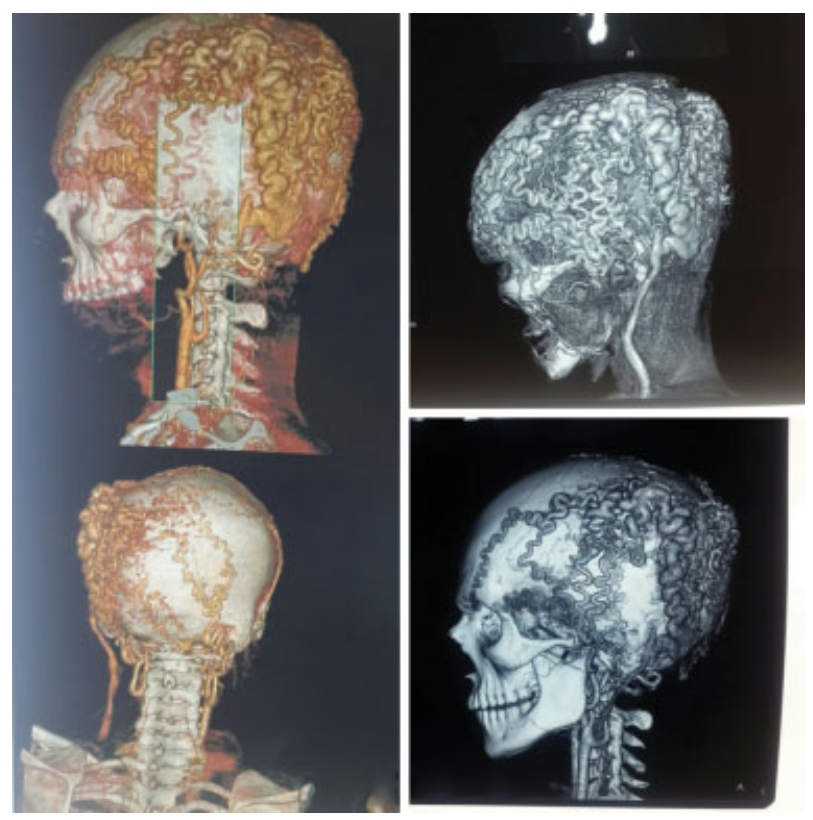

Fig. 2 Pre-op digital subtraction angiography images.
After infiltrating $2 \%$ lignocaine with adrenaline, scalp incision was made in short segments, ligating the feeding vessels and draining veins as they were encountered. Once the scalp flap was raised, the bleeding from the bone was controlled with bone wax and monopolar diathermy. After complete devascularization, the lesion along with pericranium, galea, and temporalis muscle was excised circumferentially from the subcutaneous tissue with the use of bipolar diathermy and sharp dissection without breaching the skin (-Fig. 3). Intra-op, it was very difficult to get plane of dissection due to very large tortuous venous varix. There was no significant blood loss during surgery.

Postoperatively, the patient had scalp necrosis over a small area, for which skin grafting was later performed. On microscopic examinations, the histopathological specimen contained various well-developed arteries and dilated veins in the connecting tissue. Repeat computed tomography (CT) angiogram showed complete disappearance of AVM without any remnant (-Fig. 4). There is no recurrence of fistula at the 3-month follow-up examination. The patient was advised to continue follow-up.

\section{Discussion}

Aberrant persistence of primitive arteriovenous interconnections is known as AVM. Scalp AVM are rare despite the intense vascularity of scalp and relatively high frequency of trauma to this region. ${ }^{1,2}$ The etiology of cirsoid aneurysm is still controversial. It is accepted that it may be either congenital or traumatic. Congenital was more common in large series published in the literature. ${ }^{3,4}$ In our case, traumatic etiology was the probable initiating factor. Due to the large size and complexity of the lesion, en block excision was planned.

Ligation of feeding vessels followed by resection of the lesion has occasionally met with success. Multiple treatment schemes have been described and, as yet, no standard form of therapy exists. Some of the reported treatments include radiation therapy, electrothrombosis, scalp tourniquet, scalp compression with pads and springs, direct injection with alcohol, and embolization. ${ }^{4,5}$ Surgical resection of the fistula is usually successful, as was for this patient. Direct surgical excision risks severe intraoperative blood loss.

The abnormal vascular channel dilatation over the scalp often results in deformity of the scalp that is usually not lifethreatening unless it causes hemorrhage but can lead to substantial cosmetic and social disturbances. ${ }^{5,6}$ Scalp arteriovenous fistulas are roughly evenly distributed among the frontal, temporal, and parietal regions. Occipital region scalp AVMs have been remotely reported, which is found in our present case report. The AVM feeder vessels mainly arise from the subcutaneous tissue layer of the scalp. The source of feeder arteries most frequently includes the external carotid, occipital, and supraorbital arteries. Clinical manifestations relate to the size of fistula, and patient may present with loud bruit, hemorrhage, headache, and, in severe cases, scalp necrosis. ${ }^{6}$ The lesion usually begins as small subcutaneous lump, which, over a period of time, evolves into grotesque, deforming mass. 


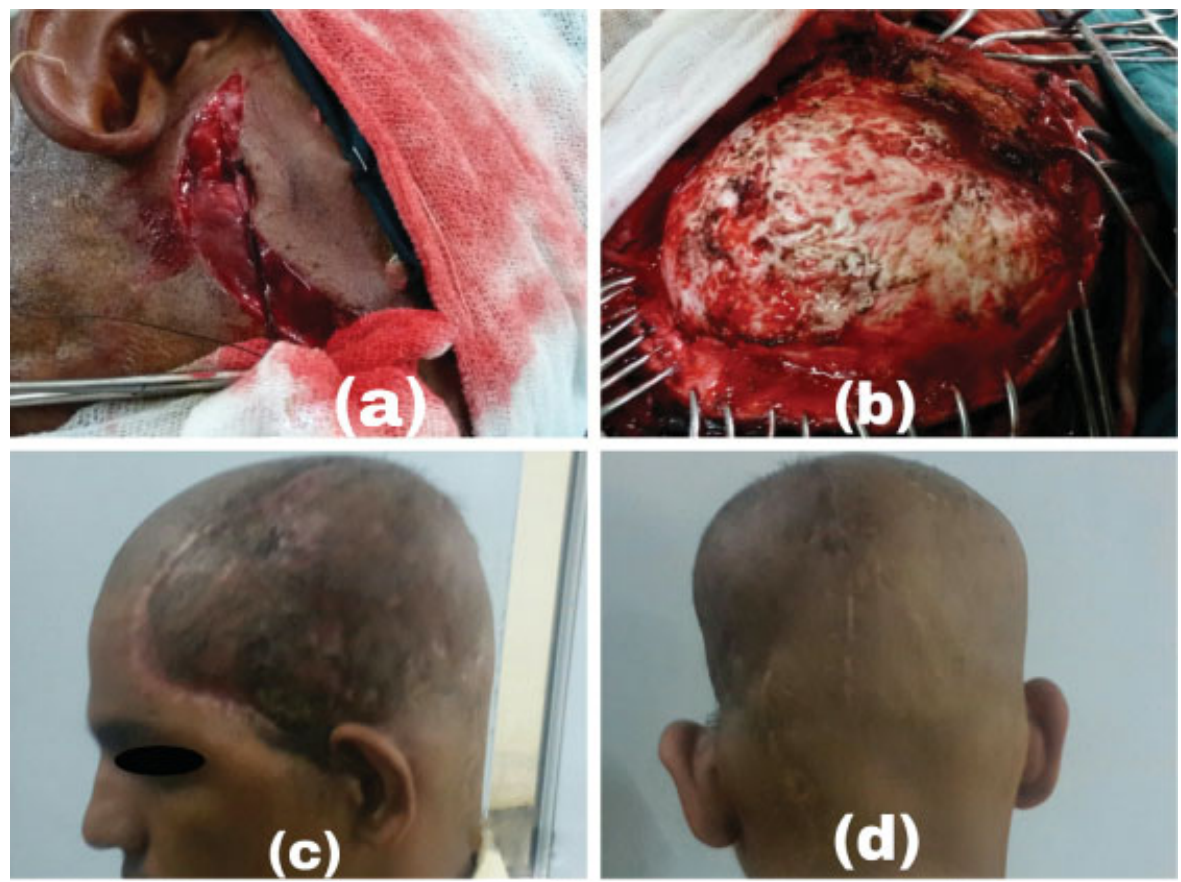

Fig. 3 Intra-op photograph before ligation of feeding arteries (a) and after en block excision of the lesion (b). Post-op photographs of the patient 2 months after surgery $(\mathrm{c}, \mathrm{d})$.

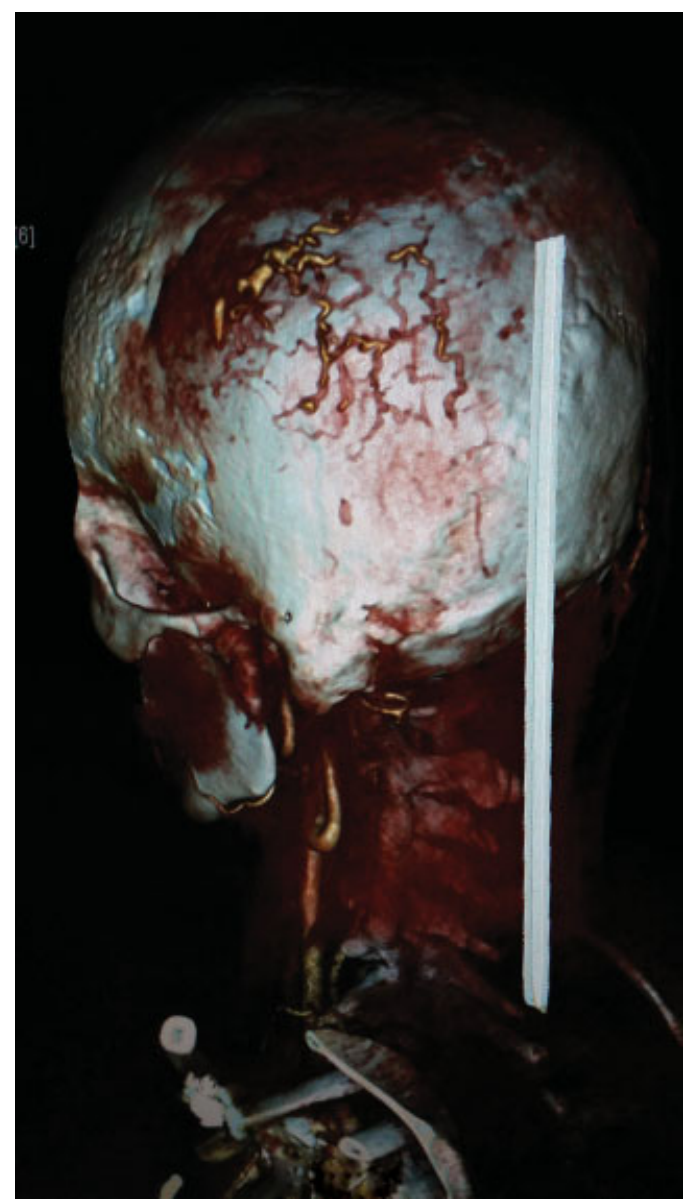

Fig. 4 Post-op CT angiography image showing resolution of lesion and no recurrence 6 months after surgery.
Digital subtraction angiography is the gold standard investigation for these lesions, which provides a road map of arterial supply and venous drainage. ${ }^{1}$ Although the lesion lies in the subcutaneous layer of scalp, the pericranial component of the lesion is of surgical importance. ${ }^{7}$ An important part of surgery is the excision of pericranial component with control of bone bleeding using bone wax. Endovascular and percutaneous occlusions of the fistulas have been described. The results of endovascular occlusion are dependent on the angioarchitecture of the fistula, the supplying arteries, and draining venous structures. Arterial approaches may not often be successful in occluding the entire fistula due to the problem of multiple feeding arteries. In our case, complex multiple feeding arteries were present, so endovascular approach was not considered. Recurrence has been reported as late as 18 years after complete surgical excision. ${ }^{8}$ Hence, regular follow-up is recommended.

\section{Conclusion}

Cirsoid aneurysm of the scalp is progressive in nature and is a rare disorder. En block surgical excision seems to be the most effective treatment with good results. During surgery, proximal control of feeding arteries, careful dissection, and removal of pericranial component and temporalis muscle are essential to prevent complications and recurrences.

\section{References}

1 Muthukumar N, Rajagopal V, Manoharan AV, Durairaj N. Surgical management of cirsoid aneurysms. Acta Neurochir (Wien) 2002; 144(4):349-356 
132 Cirsoid Aneurysm of the Scalp Barhate et al.

2 Nagasaka S, Fukushima T, Goto K, Ohjimi H, Iwabuchi S, Maehara F. Treatment of scalp arteriovenous malformation. Neurosurgery 1996;38(4):671-677, discussion 677

3 Khodadad G. Familial cirsoid aneurysm of the scalp. J Neurol Neurosurg Psychiatry 1971;34(6):664-667

4 Gurkanlar D, Gonul M, Solmaz I, Gonul E. Cirsoid aneurysms of the scalp. Neurosurg Rev 2006;29(3):208-212

5 Chatterji P, Purohit GN, Soni NK. Cirsoid aneurysm of the auricle and adjoining scalp. J Laryngol Otol 1977;91(11):997-1002
6 Barnwell SL, Halbach VV, Dowd CF, Higashida RT, Hieshima GB. Endovascular treatment of scalp arteriovenous fistulas associated with a large varix. Radiology 1989;173(2): 533-539

7 Elkin DC. Cirsoid aneurysm of the scalp: report of four cases. Ann Surg 1946;123:591-600

8 Wilkinson HA. Recurrence of vascular malformation of the scalp 18 years following excision. Case report. J Neurosurg 1971;34(3): 435-437 